\title{
Static Imaging
}

National Cancer Institute

\section{Source}

National Cancer Institute. Static Imaging. NCI Thesaurus. Code C156765.

Imaging data acquired over a single time frame. The result is a single frame that represents the average amount of radioactivity during the scan period. Only semiquantitative information can be derived from static acquisitions (e.g., relative tracer uptake). 